\title{
Le doute entrepreneurial comme facteur de changement : impact de l'accompagnement d'un mentor'
}

\author{
Etienne St-Jean \\ Professeur \\ Chaire de recherche UQTR sur la carrière entrepreneuriale \\ Institut de Recherche sur les PME, Université du Québec à Trois-Rivières \\ 3351, boul. des Forges, CP 500, 1425 Pavillon Desjardins-Hydro-Québec \\ Trois-Rivières, Qc G9A 5H7 \\ etienne.st-jean@uqtr.ca \\ Amélie Jacquemin* \\ Chercheur \\ Louvain School of Management, CRECIS/ESSCA Ecole de Management \\ 1, Place des Doyens, 1348 Louvain-la-Neuve, Belgique \\ amelie.jacquemin@uclouvain.be
}

\section{Résumé}

Cet article a pour objectif de mieux comprendre le phénomène de changement entrepreneurial qui se produit lorsqu'un entrepreneur vit une période de doute. Un changement intervient dans la mesure où une phase aigüe de doute préfigure soit l'abandon pur et simple de l'activité entrepreneuriale, soit sa redéfinition (Valéau, 2007). En effet, pour rebondir face au doute, l'entrepreneur devra reconstruire son projet en s'engageant autrement (Valéau, 2006, 2007). Partant du constat que très peu d'études s'interrogent sur les dispositifs qui permettraient d'aider l'entrepreneur à surmonter ses doutes, nous avons mené une recherche à l'aide d'un questionnaire administré auprès de 362 entrepreneurs mentorés du réseau de mentorat d'affaires de la Fondation de l'entrepreneurship au Québec. Nos résultats mettent clairement en évidence une diminution du doute chez l'entrepreneur novice qui a bénéficié de l'accompagnement d'un mentor.

\begin{abstract}
This paper aims to better understand the phenomenon of entrepreneurial change that occurs when facing a period of doubt. During periods of doubt, entrepreneur's commitment becomes more uncertain as doubt forces him to update his expectations and to see his venture in a new light (Valéau, 2007). It is often through this step that entrepreneurs rebuild the commitment needed to carry on (Valéau, 2006, 2007). However, it is not clear how various support services would help an entrepreneur to overcome his doubts. We have led an investigation of this issue based upon a questionnaire administered to 362 entrepreneurs supported by mentors affiliated to the business mentorship network of the Entrepreneurship's Foundation (Quebec). Our results clearly show a doubt's decrease among nascent mentored entrepreneurs who has benefited from a mentor support.
\end{abstract}

\footnotetext{
${ }^{1}$ St-Jean, É. et A. Jacquemin (2012), «Le doute entrepreneurial comme facteur de changement : impact d'un mentor », Revue Interdisciplinaire sur le Management, l'Humanisme et l'Entreprise, vol. 1 no. 3, p. 82-96
} 


\section{Mots-clés}

Entrepreneur - Changement - Doute - Accompagnement

\section{Key words}

Entrepreneur - Change - Doubt - Support

\section{Introduction}

En 1989, Gartner se demandait si les chercheurs en entrepreneuriat se posaient la bonne question en s'intéressant à la personne de l'entrepreneur («Qui est-il?»). Il proposait alors un déplacement du questionnement scientifique vers le processus entrepreneurial («Que fait l'entrepreneur? »). Un grand nombre de recherches ont ensuite été menées sur ce processus. L'étude des motivations qui amènent l'entrepreneur à se lancer dans l'aventure entrepreneuriale se situe au cœur de ces travaux (p. ex. Reynolds et al., (2001), Bhola et al., (2006)). La question des phases de démotivation qui peuvent traverser ce processus est, à l'inverse, très peu investiguée. On sait pourtant que le manque de temps, l'incertitude financière, la difficulté de quitter son travail et de trouver du personnel qualifié ainsi que le besoin de faire connaître son entreprise et ses produits constituent les principaux problèmes vécus dans la période du démarrage (p. ex. Lorrain et Laferté (2006) ou Schaper et al. (2005)). D'autres problèmes peuvent également surgir après cette phase de lancement et jusqu'à la cessation de l'activité entrepreneuriale. Le manque d'intérêt de la communauté scientifique pour la compréhension des phases plus difficiles du processus entrepreneurial n'est pas surprenant dans la mesure où l'image d'un entrepreneur pétri de doutes et d'incertitudes s'oppose à la figure mythique de l'entrepreneur héroïque des temps modernes (Janssen et Schmitt, 2011; Shaver, 1995)). La mise en exergue des phases de doute traversant le parcours de l'entrepreneur écorne l'image traditionnellement véhiculée d'une personne très confiante, qui a le goût du risque et qui résiste facilement à l'incertitude (Rahim, 1996; Simon et al., 2002; Simon et al., 2000). 
Ces périodes de doute engendreraient chez l'entrepreneur une remise en cause profonde de son engagement (Valéau, 2006, 2007). En effet, le doute préfigurerait un processus de changement qui pourrait, soit prendre la forme radicale de l'abandon pur et simple de l'activité entrepreneuriale, soit conduire à sa redéfinition. Seul Valéau $(2006,2007)$ semble s'être intéressé à ce phénomène de changement amorcé par le doute en l'étudiant de façon empirique et exploratoire. Nous pensons que ce phénomène mérite davantage d'attention. C'est pourquoi nous avons mené une étude de terrain sur le doute entrepreneurial avec l'objectif d'en identifier les contours, les caractéristiques, les causes, les conséquences et les acteurs. Sur ce dernier point, nous sommes convaincus de l'importance pour un entrepreneur d'être enchâssé dans des réseaux extra-organisationnels pouvant l'entourer d'une toile protectrice. C'est pourquoi, nous avons plus spécifiquement choisi d'étudier le rôle de l'accompagnement dans la gestion du doute. A cet égard, le mentorat, en offrant soutien moral, encouragements, conseils et partage de connaissances (Gravells, 2006; McGregor et Tweed, 2002; Miettinen, 2003; Wikholm et al., 2005), est susceptible d'aider les entrepreneurs novices à retrouver la motivation nécessaire, suite à leur changement de carrière, pour poursuivre le développement de leur affaire (Valéau, 2006). Avant de présenter les analyses qui nous permettront de valider l'effet du mentorat sur le doute entrepreneurial, la prochaine section abordera la notion de doute et se poursuivra avec le mentorat pour entrepreneur.

\section{Problématique}

Avant de présenter les analyses qui nous permettront de valider l'effet du mentorat sur le doute entrepreneurial, cette section aborde la notion de doute et se poursuit avec le mentorat pour entrepreneur.

\section{L'entrepreneur : un être pétri de doutes?}

Grâce à une série d'entretiens réalisés auprès d'entrepreneurs actifs en France, en Angleterre, au Québec et sur des îles de l'océan Indien, Valéau $(2006,2007)$ a mis en évidence les phases de 
doute que vivent les entrepreneurs. Ce sujet semble toutefois tabou. Nous n'avons d'ailleurs identifié aucune autre étude empirique sur ce phénomène de doute que celles de ce chercheur. Quelques auteurs ont néanmoins étudié le «capital psychologique » de l'entrepreneur et, plus particulièrement, l'aptitude de ce dernier à faire face au stress que génèrent ses activités d'affaires (p. ex. Cardon et McGrath (1999), Jensen (2008), Pareek (1994) ou Rahim (1996)). Torrès (2009, 2010) a développé, par ailleurs, une « équation fondamentale de la santé patronale » dans laquelle il met en balance les facteurs pathogènes et salutogènes pouvant exercer un impact sur la santé des dirigeants de PME. Les facteurs pathogènes qu'il cite sont la surcharge de travail, l'incertitude, le stress et la solitude. Plusieurs chercheurs ont étudié la surcharge de travail et le stress des dirigeants (Ahmad et Salim, 2009; Akande, 1994; Boyd et Gumpert, 1983; Buttner, 1992; Harris et al., 1999; McDowell-Larsen, 2007; Pareek, 1994; Rousillon et Duval-Hamel, 2006). Gumpert et Boyd (1984) ont mis en exergue l'isolement, voire la solitude, que vit la plupart des dirigeants d'entreprises. Torrès (2009) insiste, quant à lui, sur les doutes et la souffrance qu'engendre un tel isolement. Selon cet auteur, c'est ce qui explique que l'entrepreneur se tourne vers des réseaux patronaux ou des associations de pairs pour diminuer sa solitude. À cet égard, Valéau (2006) montre que le doute entrepreneurial est lié à des états psychologiques complexes variant d'un entrepreneur à l'autre.

Valéau (2007) distingue toutefois le concept de «stress » du concept de «doute » en indiquant que « le stress est une tension, une réaction physique et psychique motivante destinée à faire face au danger, alors que le doute correspond à une remise en cause plus « existentielle », à une perte de sens se traduisant par une baisse d'engagement ». Cet auteur développe un véritable modèle $\mathrm{du}$ processus d'engagement entrepreneurial et étudie le phénomène de doute qui traverse ce processus. Pour lui, l'engagement dit « de départ » est profondément remis en question lors de phases de doutes et de confusions « aigües ». Le doute surgit dès que l'entrepreneur expérimente des réalités qui ne sont pas conformes à ses attentes, à ses idéaux de départ ou lorsque l'entrepreneur est inquiet quant à ses capacités à réussir dans ses affaires (Valéau, 2006, 2007). Il doit alors adapter son engagement aux réalités rencontrées. Pour surmonter le doute, l'entrepreneur doit «réajuster» son engagement, c'est-à-dire revoir sa manière d'être entrepreneur et son projet d'affaire. La phase de doute débouchera donc très souvent sur des décisions aussi importantes que celle, par exemple, de l'abandon d'un marché ou la séparation 
d'avec un associé. De telles décisions découlent de l'actualisation des liens entre le projet personnel de l'entrepreneur et son projet d'entreprise (Bruyat, 1994; Valéau, 2007).

Nous nous tournons à présent vers la littérature sur le mentorat que nous utilisons pour développer nos hypothèses de recherche.

\section{Le mentorat : une formule d'accompagnement particulièrement adaptée à la gestion du doute?}

Selon D'Abate et al. (2003), il n'existerait pas moins de 13 formes distinctes d'accompagnement. On retrouve notamment le coaching, le counselling, la médiation, le parrainage et le mentorat dans cette «nébuleuse » des formules d'accompagnement (Paul, 2004). Nous avons choisi d'étudier l'impact du mentorat dans la gestion du doute entrepreneurial dans la mesure où la fonction ou posture dite «herméneutique » est centrale dans cette forme d'accompagnement. Il s'agit pour le mentor de mobiliser à plein sa capacité d'empathie pour véritablement «lisser l'amplitude des émotions » du porteur de projet (Verzat et Gaujard, 2009). Il doit pouvoir remonter le moral du mentoré déprimé, mais aussi amener ce dernier à relativiser ses moments d'euphorie. Cette attitude d'écoute et de protection permet au mentoré de «tenir dans la durée face aux multiples incertitudes ». Verzat et Gaujard (2009) affirment que ce sont les accompagnateurs qui ont eux-mêmes exercé une activité entrepreneuriale, et a fortiori, si celle-ci s'est soldée par un échec, qui auront le plus à cœur de prémunir le porteur de projet contre tous les risques d'échec.

Un mentor est généralement une personne possédant certaines qualités ou qui est en position d'autorité et qui veille de façon bienveillante sur un individu plus jeune, lequel bénéficie des conseils et du soutien de son mentor. Dans le contexte de l'entrepreneur, bien qu'il puisse exister plusieurs définitions, le mentorat concerne l'établissement d'une relation de soutien entre un entrepreneur novice (nommé le mentoré) et une personne expérimentée du monde des affaires (appelé le mentor), le dernier permettant au premier de se développer en tant que personne. Tel 
que le suggère Paul (2004), le mentorat se distinguerait du coaching, du tutorat ou du compagnonnage du fait qu'il est davantage orienté vers la quête de sens plutôt que l'acquisition de techniques. Sammut (1998a, b, 2003) reprend cette notion de quête de sens dans son plaidoyer pour un accompagnement "pertinent », c'est-à-dire un accompagnement permettant au créateur de libérer son talent et de s'autonomiser, au lieu de se contenter de lui offrir une formation standardisée et les connaissances de base nécessaires à la création. McGregor et Tweed (2002) ont étudié le type d'interventions pratiquées dans le cadre d'un programme de mentorat auprès d'une clientèle de femme d'affaires. En termes de soutien à la gestion, ces dernières ont déclaré avoir reçu des conseils, des pistes de solution, de l'aide relativement à plusieurs fonctions de l'entreprise (marketing, finance, etc.) et avoir aussi bénéficié du réseau de contacts de leur mentor. Au niveau davantage psychologique, elles mentionnent un impact en termes de motivation, de confiance et elles soulignent que cet accompagnement les fait sortir de leur isolement et leur permet d'obtenir de la rétroaction. Le fait de bénéficier de tout ce soutien aurait aussi un impact positif sur le chiffre d'affaires de leur entreprise. D'autres recherches ont confirmé l'existence de bénéfices similaires en termes de soutien à la gestion de l'entreprise (Bisk, 2002; Cull, 2006; Kent et al., 2003), de soutien davantage psychologique (Nandram, 2003; Waters et al., 2002) et même à ces deux niveaux simultanément (Gravells, 2006; Miettinen, 2003; St-Jean et Audet, 2012; Wikholm et al., 2005).

Évidemment, de telles retombées seraient susceptibles de réduire les périodes de doute à l'égard du projet d'affaire. Que ce soit en améliorant les compétences de l'entrepreneur ou en le faisant se sentir plus confiant en lui (p. ex. St-Jean et Audet (2012)), il pourrait envisager la poursuite de sa carrière d'entrepreneur avec plus d'enthousiasme et d'optimisme. En outre, puisque le mentorat est davantage orienté vers la quête de sens pour le mentoré (p. ex. Paul (2004)) et que c'est justement ce qui permet à l'entrepreneur vivant une période de doute de redéfinir son projet (Valéau, 2007), cette formule serait alors toute indiquée pour l'entrepreneur. Ces considérations nous amènent à développer la première hypothèse de notre recherche de la manière suivante :

H1 : Le mentorat réduit le doute de l'entrepreneur à l'égard de son projet d'affaires. 
Les relations de mentorat ne sont pas toutes équivalentes. Au-delà de la durée ou de la fréquence des rencontres qui peuvent en influencer la qualité (p. ex. Wanberg et al. (2006)), les caractéristiques du mentor et du mentoré, tout comme la nature de la relation qui se développe, sont également importantes à ce niveau (Allen et Eby, 2008; Poteat et al., 2009; Wanberg et al., 2003). Ainsi, la «chimie » entre le mentor et son mentoré semble essentielle au succès d'une relation de mentorat de l'entrepreneur (Couteret et al., 2006; Cull, 2006). Cette « chimie » n'est pas en lien avec le niveau de compétence du mentor. Par exemple, des mentors ont eu des relations où la « chimie » initiale ne s'est pas produite avec un mentoré mais ont eu des relations satisfaisantes avec d'autres mentorés subséquemment (Gravells, 2006). Aussi, comme le propose Kram (1985), la confiance est une composante essentielle dans la relation de mentorat afin de rehausser la qualité et l'efficacité de la relation. Cette constatation trouve aussi écho dans un contexte de mentorat de l'entrepreneur (Cull, 2006).

En plus de la confiance et pour l'aider à la bâtir, certains auteurs insistent sur le rôle joué par la similitude perçue entre les membres de la dyade (Allen et Eby, 2003; Ensher et Murphy, 1997; Wanberg et al., 2006). Selon Dalley et Hamilton (2000), certaines différences quant à la culture des membres de la dyade, notamment en ce qui concerne la façon de gérer une entreprise et d'obtenir du succès, peut mener la relation de mentorat à un échec (Dalley et Hamilton, 2000). Dans le cas des entrepreneurs, ce serait plutôt la carrière du mentor qui pourrait avoir un impact au niveau de la similitude démographique. En effet, le mentor qui a été lui-même en affaires en tant qu'entrepreneur pourrait témoigner des difficultés inhérentes de la carrière, en stipulant que ces difficultés sont souvent passagères, et transmettre de manière plus efficace la culture entrepreneuriale. À cet effet, d'autres recherches indiquent que la fonction de modèle de rôle du mentor est plus marquée chez les mentors ayant été en affaires que ceux n'ayant jamais été propriétaires d'entreprise (St-Jean, 2011). En outre, Valéau (2006) montre clairement que la réduction du doute n'aura pas lieu si le doute s'accompagne d'une dégradation du degré de confiance que l'entrepreneur place en son accompagnateur. À cet égard, il démontre que le fait de connaître une autre personne ayant créé son entreprise permet à l'entrepreneur de se rendre compte que le doute est présent dans toute aventure entrepreneuriale et, dès lors, relativiser les difficultés qu'il rencontre. 
Ces résultats se focalisent sur le degré de confiance entre les membres de la dyade « entrepreneur-accompagnateur » en période de doute en soulignant l'effet modérateur joué par le fait que le mentoré connaisse un tiers entrepreneur. Ils ne prennent pas en considération l'impact du degré de similitude perçue entre les membres de la dyade. À cet égard, le fait que l'accompagnateur ait lui-même une expérience entrepreneuriale pourrait avoir une influence en période de doute. Nous pouvons penser que non seulement la confiance et la similitude perçue ainsi que la carrière entrepreneuriale du mentor auront un effet positif sur la réduction du doute chez le mentoré, mais également qu'un mentor avec une carrière d'entrepreneur permettra une meilleure réduction du doute lorsque la confiance et la similitude perçue sont faibles. Toutes ces considérations amènent les hypothèses suivantes :

- H2 : Le mentor entrepreneur réduira davantage le doute chez le mentoré qu'un mentor n'ayant pas été entrepreneur.

- H3 : Un niveau élevé de confiance et de similitude perçue dans la relation de mentorat permettent de réduire davantage le doute chez le mentoré qu'un niveau faible.

- H4 : Dans les relations de mentorat ayant un niveau de confiance et de similitude perçue faible, la réduction du doute sera plus grande lorsque le mentor a une carrière d'entrepreneur.

Avant de développer notre méthodologie de recherche, nous présentons à présent notre terrain de recherche.

\section{Un terrain de recherche proposé par la Fondation de l'entrepreneurship du Québec}

Le Réseau $\mathrm{M}$, un réseau de mentorat d'affaires, qui sert de toile de fond à la présente recherche, a été créé en 2000 par la Fondation de l'entrepreneurship, un organisme voué au développement économique sur le territoire du Québec. Il est offert aux entrepreneurs novices par le biais d'environs 70 cellules de mentorat dispersées sur l'ensemble du territoire du Québec. Ces cellules 
sont généralement soutenues par des organismes de développement économique tels les Centres locaux de développement (CLD), les Sociétés d'aide au développement des collectivités (SADC) et les chambres de commerce locales. Ces organismes assurent le développement du programme au niveau local ou régional, tout en adhérant au modèle du mentorat d'affaires développé par la Fondation.

\section{Méthodologie de recherche}

Dans cette section, nous présentons notre questionnaire de recherche, les mesures que nous avons réalisées et notre méthode d'analyse.

\subsection{Le questionnaire de recherche}

Nous avons administré un questionnaire auprès de 362 mentorés affiliés au réseau de mentorat d'affaires de la Fondation de l'entrepreneurship. Ce chiffre correspond à un taux de réponse de $36,9 \%$. Ce questionnaire nous a permis de mesurer le niveau de doute passé, le niveau de doute actuel, le changement du niveau de doute, ainsi que le niveau de similitude et de confiance perçue au sein de la dyade mentor-mentoré. Ces mesures sont présentées ci-dessous.

\subsection{Les mesures}

Pour mesurer la "période de doute passée », dans les débuts du questionnaire, les répondants devaient évaluer leur niveau d'accord à propos des affirmations suivantes : "Avant ma relation de mentorat... 1- J'ai déjà pensé abandonner mon projet d'affaires, 2- J'ai vécu des moments de découragement face à mon projet d'affaires, 3- Mon entreprise était parfois comme un boulet accroché à mes pieds, et 4- J'ai envisagé de devenir salarié pour une autre organisation. Pour la "période de doute actuelle », nous avons utilisé les mêmes items mais cette fois-ci, formulés au présent (p. ex. 1-Je pense à abandonner mon projet d'affaires, etc.). Cette deuxième question sur 
le doute arrivait vers la fin du questionnaire, pour laisser le temps au répondant d'oublier ses premières réponses, et elle était précédée de la demande suivante : "Vous devez penser à la manière dont vous vous sentez aujourd'hui ».

Nous avons calculé le «changement dans le niveau de doute » à l'égard du projet d'affaires en soustrayant le niveau de doute actuel (somme des items) du niveau de doute au moment de solliciter l'aide d'un mentor (somme des items).

La mesure de "similitude perçue » avec le mentor a été développée en s'inspirant de celle proposée par Allen et Eby (2003), laquelle vérifie 1-la similitude des valeurs, 2-des intérêts et 3de la personnalité. Nous avons aussi ajouté l'item proposé par Ensher et Murphy (1997) à propos de la similitude des points de vue (4).

La mesure de la «confiance envers le mentor » a été créée spécialement pour cette recherche, en se basant sur les trois composantes de la confiance envers une autre personne de Rempel et Holmes (1986), soit 1-la fiabilité, 2-la prévisibilité et 3-la confiance.

\subsection{La méthode d'analyse}

Pour s'assurer que l'effet du mentorat ait pu se faire sentir chez le mentoré (Kram, 1983), nous avons sélectionné seulement les relations (250 sur 362) qui ont duré six mois ou plus. Étant donné que les rencontres ont lieu en moyenne une fois par mois et que ce programme de mentorat formel jumelle officiellement la dyade pour une année, bien que plusieurs relations continuent après cette période, nous pensons qu'une période de six mois pourrait être suffisante pour obtenir l'effet escompté. 


\section{Encadré 1: Échantillon retenu pour l'analyse}

L'échantillon contient 162 hommes $(51,6 \%)$ et 152 femmes $(48,4 \%)$. Ces derniers ont été jumelés à 275 mentors hommes $(81,4 \%)$ et à 63 mentors femmes $(18,6 \%)$. Les mentorés sont assez scolarisés puisque 173 (55\%) d'entre eux ont au moins un diplôme universitaire. Ils ont 39,8 ans en moyenne et l'âge varie entre 23 et 70 ans. Au moment du démarrage, 24\% n'avaient aucune expérience du secteur d'activité de leur entreprise, $33,2 \%$ moins d'un an, 46,2\% moins de trois ans et 61,6\% moins de cinq ans. Au niveau de l'expérience en affaires au démarrage, la majorité $(51,1 \%)$ n'avait aucune expérience, $63,4 \%$ avaient moins d'un an, 73,6\% avaient moins de trois ans et $82,9 \%$ avaient moins de cinq ans. La presque totalité des mentorés avaient une entreprise active au moment du jumelage (293 sur 314, 93,3\%) et les autres étaient en processus de démarrage. Celle-ci avait peu d'employés, avec une moyenne de 4,48. Les secteurs industriels sont variés, mais avec une légère concentration dans les services professionnels (62, pour 23,0\%), dans la fabrication $(39$, pour $14,4 \%$ ) et dans le commerce de détail (32, pour 11,9\%). Les relations de mentorat ont duré en moyenne 16,07 mois. La majorité des répondants étaient encore en relation de mentorat au moment de répondre $(58,6 \%)$.

\section{Résultats}

Dans cette section, nous nous interrogeons successivement sur trois éléments. Nous nous demandons tout d'abord si le mentorat réduit le doute de l'entrepreneur. Ensuite, nous nous interrogeons sur l'influence de la carrière du mentor sur la réduction du doute entrepreneurial. Enfin, nous nous demandons si la qualité de la relation de mentorat a une influence dans la réduction du doute entrepreneurial.

\subsection{Le mentorat réduit-il le doute?}


Nous avons d'abord fait une analyse de différence de moyenne pour échantillon appariés afin de vérifier si le mentorat permet l'amélioration du niveau de doute à l'égard du projet. Le Tableau 1 confirme une amélioration significative de la moyenne du doute entre le début de la relation et au moment de répondre au questionnaire. Tel que prévu, cela confirme H1.

Tableau 1: Test $\mathrm{t}$ de l'amélioration du doute $(\mathrm{n}=250)$

\begin{tabular}{ccc}
\hline $\begin{array}{c}\text { Période de doute passée } \\
3,47\end{array}$ & Période de doute actuelle & Sig. \\
& 2,78 & $0,000^{* * *}$ \\
\hline$* * *=\mathrm{p} \leq 0.001 \quad * *=\mathrm{p} \leq 0.01$ & $*=\mathrm{p} \leq 0.05$ &
\end{tabular}

Cette réduction du doute n'est pas surprenante dans la mesure où l'on sait que le mentorat peut générer des retombées positives pour l'entrepreneur aussi diverses que la réduction de la solitude et de l'isolement (McGregor et Tweed, 2002; St-Jean et al., 2007), un soutien moral (Miettinen, 2003) ou la préservation de la motivation (Wikholm et al., 2005). D'autres auteurs soulignent également l'existence des retombées pour l'entreprise (Deakins et al., 1998; Sullivan, 2000), ce qui pourrait également engendrer la réduction du doute à l'égard du projet d'affaires. Les résultats obtenus ici permettent de confirmer l'utilité du mentorat pour réduire le doute.

\subsection{La carrière du mentor a-t-elle une influence?}

Par la suite, nous avons comparé l'amélioration du doute selon la carrière du mentor. Le Tableau 2 montre que l'accompagnement tant d'un mentor entrepreneur que d'un mentor qui a eu une autre carrière (par exemple, des retraités du gouvernement, des cadres d'institutions financières, etc.) permet de réduire le doute chez le mentoré de manière significative. Par contre, si le niveau de doute passé (à savoir au moment de demander l'aide du mentor) est similaire pour les deux catégories de mentor, le niveau de doute après la relation est significativement plus bas chez les mentors entrepreneurs, ce qui va dans le sens de $\mathrm{H} 2$. 
Tableau 2: Comparaison du doute passé et actuel selon la carrière du mentor

\begin{tabular}{lccc}
\hline & $\begin{array}{c}\text { Période de doute } \\
\text { passée }\end{array}$ & $\begin{array}{c}\text { Période de doute } \\
\text { actuelle }\end{array}$ & $\begin{array}{c}\text { Test } t \text { apparié } \\
(\text { Sig. })\end{array}$ \\
\hline Mentor entrepreneur $(\mathrm{n}=118)$ & 3,33 & 2,54 & $0,000^{* * *}$ \\
Mentor non entrepreneur $(\mathrm{n}=132)$ & 3,59 & 2,99 & $0,000^{* * *}$ \\
\hline Test $t$ (Sig.) & 0,260 & $0,016^{*}$ & \\
\hline$* * *=\mathrm{p} \leq 0.001 \quad * *=\mathrm{p} \leq 0.01 \quad *=\mathrm{p} \leq 0.05$ & &
\end{tabular}

Toutefois, la comparaison de la réduction du doute (c'est-à-dire le doute passé moins le doute actuel) selon la carrière entrepreneuriale ou non du mentor ne donne pas lieu à un résultat statistiquement significatif (voir Tableau 3). H2 n'est donc que partiellement confirmée.

Tableau 3 : Comparaison de l'amélioration du doute selon la carrière du mentor $(\mathrm{n}=232)$

\begin{tabular}{|c|c|c|}
\hline Mentor entrepreneur & Mentor non entrepreneur & Sig. \\
\hline 3,60 & 2,62 & 0,256 \\
\hline
\end{tabular}

Ces résultats montrent que, toute chose étant égale par ailleurs, le doute pouvait être réduit significativement par tous les types de mentors, qu'ils aient été en affaires ou non. Bien que le doute actuel soit significativement moins grand lorsque le mentor est entrepreneur, la réduction du doute n'est pas davantage prononcée pour ceux-ci, ce qui démontre que tous les mentors sont relativement capables d'agir sur cette dimension.

\subsection{La qualité de la relation de mentorat a-t-elle une influence?}


Nous avons séparé les mentorés selon le niveau de confiance et de similitude perçue à l'égard du mentor. Nous avons utilisé la médiane pour créer des groupes, en regroupant ceux dont la confiance et la similitude perçue est inférieure à la médiane et les autres dont ces deux dimensions étaient supérieures ou égales à la médiane. Des relations ont toutefois été exclues puisque certains mentorés avaient un niveau de confiance inférieur à la médiane mais une similitude perçue supérieure à la médiane, ou l'inverse. Comme nous pouvons le constater au Tableau 4, les groupes ayant une confiance et une similitude perçue relativement basses ont réduit leur doute autant que ceux dont ces dimensions sont élevées. Ce résultat ne permet donc pas de valider $\mathrm{H} 3$.

Tableau 4 : Comparaison du doute passé et actuel selon la qualité de la relation de mentorat

\begin{tabular}{lccc}
\hline & $\begin{array}{c}\text { Période de } \\
\text { doute passée }\end{array}$ & $\begin{array}{c}\text { Période de } \\
\text { doute actuelle }\end{array}$ & $\begin{array}{c}\text { Test } t \\
\text { apparié (Sig.) }\end{array}$ \\
\hline Confiance et similitude $<$ médiane $(\mathrm{n}=60)$ & 3,58 & 2,80 & $0,001^{* * *}$ \\
Confiance et similitude $\geq$ médiane $(\mathrm{n}=103)$ & 3,25 & 2,64 & $0,002^{* *}$ \\
\hline$* * *=\mathrm{p} \leq 0.001 \quad * *=\mathrm{p} \leq 0.01 \quad *=\mathrm{p} \leq 0.05$ & & &
\end{tabular}

Finalement, nous nous sommes demandés si la carrière entrepreneuriale du mentor ne pouvait pas compenser la faible qualité de la relation de mentorat. Cette intuition s'est avérée fondée dans la mesure où nous avons identifié un effet d'interaction entre la qualité de la relation de mentorat et la carrière du mentor dans la réduction du doute. En utilisant la mesure de réduction du doute, on constate que lorsque la confiance et la similitude sont élevées, la carrière du mentor n'a pas d'effet, les mentorés réduisant de manière similaire leur doute (voir Tableau 5). Par contre, lorsque la confiance et la similitude perçue sont faibles, la carrière du mentor est importante : ce sont ceux qui ont été entrepreneurs qui font réduire davantage le doute chez les entrepreneurs novices. Ces résultats confirment $\mathrm{H} 4$. 
Tableau 5: Amélioration du doute selon la carrière du mentor et la qualité de la relation de mentorat

\begin{tabular}{lcccc}
\hline & $\begin{array}{c}\text { Mentor } \\
\text { entrepreneur }\end{array}$ & $\begin{array}{c}\text { Mentor non } \\
\text { entrepreneur }\end{array}$ & $\begin{array}{c}\text { Test } t \\
\text { (Sig.) }\end{array}$ \\
\hline Confiance et similitude $<$ médiane $(\mathrm{n}=56)$ & 5,04 & 1,23 & $0,04 *$ \\
Confiance et similitude $\geq$ médiane $(\mathrm{n}=94)$ & 3,24 & 2,69 & 0,72 \\
\hline$* * *=\mathrm{p} \leq 0.001$ & $* *=\mathrm{p} \leq 0.01$ & $*=\mathrm{p} \leq 0.05$ & 0,23 & 0,44 \\
\end{tabular}

En d'autres termes, lorsque la relation de mentorat est de grande qualité (niveaux élevés de confiance et de similitude perçue), la carrière du mentor n'a pas d'effet dans la réduction du doute. Par contre, lorsque la qualité de la relation est faible, les mentors entrepreneurs réduiront davantage le doute chez l'entrepreneur novice que ceux ayant été dans une autre carrière. Nous pensons que la fonction de modèle de rôle des mentors, qui se déploie de manière plus marquée chez les mentors entrepreneurs, pourrait être en cause (St-Jean, 2011). Par exemple, nous savons que l'apprentissage qui découle d'une relation de mentorat permet au mentoré d'obtenir de nouvelles informations susceptibles d'outrepasser les limites à son manque d'expérience, comme l'évoquaient Ucbasaran et al. (2009). Les schémas cognitifs du mentor entrepreneur, beaucoup plus complets que ceux du mentoré (p. ex. Baron, (2006)), sont partagés à ce dernier lors des discussions, ce qui lui offre l'occasion d'affiner ses propres schémas cognitifs menant à l'identification d'opportunités (St-Jean et Tremblay, 2011). Tout cela n'est possible que lorsque le mentor possède de l'expérience en affaires. Dans ce contexte, les transformations que le mentor peut induire chez le mentoré pourraient réduire le doute, malgré une relation de moins grande qualité avec le mentoré. Les fonctions psychologiques et reliées à la carrière, qui sont identiques chez les mentors entrepreneurs et les autres (St-Jean, 2011), pourraient se déployer pour réduire le doute, peu importe la qualité de la relation.

Nous nous tournons à présent vers les limites de notre analyse. 


\section{Les limites de notre étude}

L'une des limites principales à cette recherche est qu'elle n'est pas longitudinale. Nous avons toutefois tenté d'augmenter la validité de notre démarche en questionnant le répondant en début de questionnaire sur son niveau de doute au moment du jumelage (items formulés au passé), puis, en fin de questionnaire, sur son niveau de doute actuel (items formulés au présent). La difficulté réside dans le fait que le répondant doit se remémorer son niveau de doute passé, datant parfois de plusieurs années. Il est probable que certains biais liés à la remémoration des faits puissent influencer les réponses obtenues et, du coup, réduisent la validité de nos résultats (p. ex. Glick et al., (1995); Wolfe et Jackson (1987)). Valéau (2007) apporte néanmoins un éclairage intéressant sur ce point en précisant que le risque de remémoration, et donc de reconstruction de l'expérience vécue, ne pose pas tant problème lorsque le chercheur s'intéresse davantage à ce qu'un individu a $\mathrm{pu}$ vivre et ressentir qu'aux évènements en eux-mêmes. Ainsi, demander au mentoré de se remémorer son niveau de doute passé et de le comparer à son niveau de doute actuel permet au moins à celui-ci d'admettre et donc de mesurer peut-être plus justement certaines difficultés rencontrées.

Par ailleurs, la création de groupes selon les niveaux de confiance et de similitude perçue constitue également une limite à notre étude. Notre comparaison de l'importance de la réduction de doute selon un niveau bas ou un niveau élevé de confiance et de similitude perçue n'a pas été concluante. Peu importe la qualité de la relation au niveau de la confiance et de la similitude perçue, une réduction du doute se produit. Ce résultat doit être nuancé car nous pensons qu'il peut être biaisé par notre méthode d'analyse. En effet, nous n'avons retenu pour notre étude que les relations de mentorat existant depuis plus de 6 mois. Pour qu'une relation de mentorat se poursuive au-delà de 6 mois, il faut un minimum de confiance et de similitude perçue, sans quoi la relation se terminerait rapidement (p. ex. Kram (1985)). D’autres éléments de nos résultats non repris dans cet article démontrent d'ailleurs une augmentation significative de la confiance après 6 mois. Il est probable que cette situation réduise la possibilité de constater l'effet de la qualité de la relation sur la réduction du doute. Le filtre des 6 mois minimum de relation pourrait aboutir à ne conserver que des relations où la qualité est suffisamment grande pour réduire le doute. 
Séparer les groupes à la médiane polarise les extrêmes. Toutefois, les deux groupes ainsi formés pourraient être tous les deux au-delà d'un seuil minimal de confiance et de similitude perçue. Voilà qui réduit la possibilité de constater l'effet d'un manque de qualité de la relation dans la réduction du doute.

\section{Conclusions et pistes pour de futures recherches}

L'entrepreneur se trouve au cœur du processus de changement qu'induit une période de doute. Toutefois, d'autres acteurs peuvent jouer un rôle important pour aider l'entrepreneur à gérer celles-ci. À cet égard, nous avons montré que l'accompagnement d'un mentor permet de réduire le doute entrepreneurial de manière significative. Ce résultat ne nous surprend guère dans la mesure où le mentorat est une formule d'accompagnement dans laquelle la fonction herméneutique est centrale. Il s'agit d'une attitude d'écoute et de protection davantage orientée vers la quête de sens plutôt que l'acquisition de techniques (Paul, 2004). Ce sont donc moins des compétences techniques que des éléments de soutien davantage psychologiques dont a besoin l'entrepreneur pour surmonter ses doutes. Par ailleurs, le fait de bénéficié d'un soutien d'un mentor ayant lui-même été en affaires peut être également important. Toutefois, nos résultats montrent que cette fonction de modèle de rôle est significativement importante pour la réduction du doute dans le seul cas où la relation entre mentor et mentoré n'est pas de grande qualité (niveau de confiance et de similitude perçue plus faible).

Pour les organismes qui offrent des programmes de mentorat aux entrepreneurs novices, les résultats obtenus sont intéressants à plusieurs égards. D'abord, dans un contexte où plusieurs options d'accompagnement peuvent être disponibles pour un entrepreneur qui semble manifester un doute face à son engagement entrepreneurial, les responsables de structure peuvent maintenant suggérer le mentorat comme étant une formule adaptée et pertinente pour répondre à ce besoin particulier et complexe. De plus, il faut rappeler que le doute entrepreneurial peut être réduit par

tous les mentors dans la mesure où ceux-ci peuvent rapidement établir un lien de confiance avec l'entrepreneur novice et que ce dernier se perçoive de manière similaire à son mentor en termes de personnalité, de valeurs et d'intérêt; autrement dit, que la «chimie » passe entre eux. Pour y 
parvenir, une sélection des mentors basée sur les motivations qu'ils ont à devenir des bénévoles (St-Jean et El Agy, 2012) ou de la formation sur la manière d'établir une relation efficace (StJean et Mitrano-Méda, 2012) sont des options à considérer. Toujours dans la perspective de réduire le doute de l'entrepreneur, en l'absence de qualités appropriées du mentor (innées ou développées) qui permettent de développer la confiance et la similitude perçue, un entrepreneur d'expérience pourrait compenser pour ces faiblesses. Ainsi, bien qu'une sélection adéquate et une formation soient pertinentes, en l'absence de celles-ci, l'expérience entrepreneuriale pourrait être suffisante pour réduire le doute.

Pour réellement percer la «boîte noire » du phénomène de changement entrepreneurial induit par le doute, de futures recherches devront certainement tenir compte davantage de la grande diversité des origines du doute. Le doute peut être causé par des éléments aussi divers que la déception engendrée par des expériences vécues par l'entrepreneur ou les inquiétudes de l'entrepreneur concernant ses propres capacités entrepreneuriales (Valéau, 2006, 2007). Notre mesure du doute ne permet pas de capturer cette diversité puisque nous avons étudié le doute en général. Des recherches complémentaires sont dès lors requises pour affiner nos résultats. Il serait notamment intéressant de distinguer les différentes causes possibles du doute et de déterminer pour chaque cas de figure quelles sont les retombées du mentorat les plus susceptibles de provoquer la réduction du doute. Ainsi, lorsque le doute est lié aux croyances de l'entrepreneur en ses propres capacités (notion d'auto-efficacité), il serait utile de déterminer si et dans quelle mesure l'auto-efficacité de l'entrepreneur a été améliorée à l'issue de l'accompagnement d'un mentor durant la phase de doute. Par ailleurs, il pourrait être intéressant d'étudier l'impact du mentorat dans la gestion du doute en distinguant les différentes phases de la relation de mentorat et de faire le lien avec les différentes fonctions que le mentor peut assurer (p. ex. Kram, (1983, 1985)). Cela permettrait de mieux tenir compte de l'évolution de la relation de mentorat et des fonctions assurées par le mentor durant les différentes phases de cette relation et de l'influence de ces fonctions sur la gestion du doute. Enfin, l'étude de ce processus de changement nécessite de mener des mesures du niveau de doute à différents moments du temps. De futures études dans ce domaine devront dès lors être longitudinales. 


\section{Bibliographie}

Ahmad, S. Z. et F. A. A. Salim (2009), Sources of stress and the coping mechanism for Malaysian entrepreneurs, African Journal of Business Management, vol. 3, nº 6, p. 311316.

Akande, A. (1994), Coping with entrepreneurial stress: evidence from Nigeria, Journal of Small Business Management, vol. 32, $\mathrm{n}^{\mathrm{o}}$ 1, p. 83-88.

Allen, T. D. et L. T. Eby (2003), Relationship Effectiveness for Mentors: Factors Associated with Learning and Quality, Journal of Management, vol. 29, nº 4, p. 469-486.

Allen, T. D. et L. T. Eby (2008), Mentor commitment in formal mentoring relationships, Journal of Vocational Behavior, vol. 72, n 3, p. 309-316.

Baron, R. A. (2006), Opportunity Recognition as Pattern Recognition: How Entrepreneurs "Connect the Dots" to Identify New Business Opportunities, Academy of Management Perspectives, vol. 20, n 1, p. 104-119.

Bhola, R., I. Verheul, A. Thurik et I. Grilo (2006), Explaining engagement levels of opportunity and necessity entrepreneurs, EIM Business and Policy Research no ${ }^{\circ} \mathrm{H} 200610$, Erasmus School of Economics (ESE).

Bisk, L. (2002), Formal entrepreneurial mentoring: the efficacy of third party managed programs, Career Development International, vol. 7, $\mathrm{n}^{\circ}$ 5, p. 262-270.

Boyd, D. P. et D. E. Gumpert (1983), Coping with entrepreneurial stress, Leadership Organization Development Journal, vol. March-April, n 2, p. 44-64.

Bruyat, C. (1994), Contributions épistémologiques au domaine de l'entrepreneuriat, Revue française de Gestion, no 101, p. 87-99.

Buttner, E. H. (1992), Entrepreneurial stress: Is it hazardous to your health, Journal of Managerial issues, vol. 4, $\mathrm{n}^{\mathrm{o}}$ 2, p. 223-240.

Cardon, M. S. et R. McGrath (1999), When the going gets tough... Toward a psychology of entrepreneurial failure and re-motivation, Frontiers of entrepreneurship research, Babson College Entrepreneurship Research Conference, p. 58-71.

Couteret, P., E. St-Jean et J. Audet (2006), Le mentorat : conditions de réussite de ce mode d'accompagnement de l'entrepreneur, $23^{\text {ème }}$ conférence du CCPME/CCSBE, TroisRivières, Québec, 28-30 septembre.

Cull, J. (2006), Mentoring Young Entrepreneurs: What Leads to Success?, International Journal of Evidence Based Coaching and Mentoring, vol. 4, no 2, p. 8-18.

D'Abate, C. P., E. R. Eddy et S. I. Tannenbaum (2003), What's in a Name? A Literature-Based Approach to Understanding Mentoring, Coaching, and Other Constructs That Describe Developmental Interactions, Human Resource Development Review, vol. 2, no 4, p. 360384.

Dalley, J. et B. Hamilton (2000), Knowledge, Context and Learning in the Small Business, International Small Business Journal, vol. 18, no 3, p. 51-59.

Deakins, D., L. Graham, R. Sullivan et G. Whittam (1998), New venture support: an analysis of mentoring support for new and early stage entrepreneurs, Journal of Small Business and Enterprise Development, vol. 5, $\mathrm{n}^{\mathrm{o}}$ 2, p. 151-161.

Ensher, E. A. et S. E. Murphy (1997), Effects of Race, Gender, Perceived Similarity, and Contact on Mentor Relationships, Journal of Vocational Behavior, vol. 50, n 3, p. 460-481.

Gartner, W. B. (1989), "Who Is an Entrepreneur ?" Is the Wrong Question, Entrepreneurship Theory and Practice, vol. 13, n 4, p. 47-68. 
Glick, W. H., G. P. Huber, C. C. Miller, D. H. Doty et K. M. Sutcliffe (1995), Studying Changes in Organizational Design and Effectiveness - Retrospective Event Histories and Periodic Assessments, in G. P. Huber et A. H. Van de Ven (éds.), Longitudinal Field Research Methods - Studying Processes of Organizational Change, Thousand Oaks, Sage Publications.

Gravells, J. (2006), Mentoring start-up entrepreneurs in the East Midlands - Troubleshooters and Trusted Friends, The International Journal of Mentoring and Coaching, vol. 4, $\mathrm{n}^{\circ} 2$.

Gumpert, D. E. et D. P. Boyd (1984), The loneliness of the small-business owner, Harvard Business Review, vol. 62, no 6, p. 18-24.

Harris, J. A., R. Saltstone et M. Fraboni (1999), An evaluation of the job stress questionnaire with a sample of entrepreneurs, Journal of Business and Psychology, vol. 13, nº 3, p. 447455.

Janssen, F. et C. Schmitt (2011), L'entrepreneur, héros des temps modernes ? Pour une analyse critique de l'entrepreneuriat, in L. Taskin et M. De Nanteuil (éds.), Perspectives critiques en management, Bruxelles, De Boeck.

Jensen, S. M. (2008), Psychological capital and entrepreneurial stress: propositions for study, United States Association for Small Business and Entrepreneurship (USASBE), San Antonio, USA, Jan. $10^{\text {th }}-13^{\text {th }}$.

Kent, T., C. Dennis et S. Tanton (2003), An evaluation of mentoring for SME retailers, International Journal of Retail \& Distribution Management, vol. 31, nº 8/9, p. 440-448.

Kram, K. E. (1983), Phases of the Mentor Relationship, Academy of Management Journal, vol. 26, no 4, p. 608-625.

Kram, K. E. (1985), Mentoring at Work: Developmental Relationships in Organizational Life Glenview, Ill., Scott Foresman.

Lorrain, J. et S. Laferté (2006), Support Needs of the Young Entrepreneur, Journal of Small Business and Entrepreneurship, vol. 19, n 1, p. 37-48.

McDowell-Larsen, S. (2007), L'effet néfaste du stress sur les dirigeants in F. Bournois, J. DuvalHamel, J. L. Scaringella, et al. (éds.), Comités exécutifs: Voyage au cour de la dirigeance, Éditions Eyrolles.

McGregor, J. et D. Tweed (2002), Profiling a New Generation of Female Small Business Owners in New Zealand: Networking, Mentoring and Growth, Gender, Work and Organization, vol. $9, \mathrm{n}^{\mathrm{o}} 4$, p. 420-438.

Miettinen, A. (2003), Mentoring for Entrepreneurs as Educational Intervention, Internationalizing Entrepreneurship Education and Training Conference, Grenoble, France, Sept., 8-10.

Nandram, S. S. (2003), Entrepreneurs' Need For Mentoring And Their Individual Differences, ICSB $48^{\text {th }}$ World Conference, North Ireland, June, 15-18.

Pareek, U. (1994), Entrepreneurial role stress, Journal of Entrepreneurship, vol. 3, no 1, p. 55-67.

Paul, M. (2004), L'accompagnement : une posture professionnelle spécifique, Paris, L'Harmattan.

Poteat, L., K. Shockley et T. Allen (2009), Mentor-protégé commitment fit and relationship satisfaction in academic mentoring, Journal of vocational behavior, vol. 74, $\mathrm{n}^{\circ} 3$, p. 332337.

Rahim, A. (1996), Stress, Strain, and Their Moderators: An Empirical Comparison of Entrepreneurs and Managers, Journal of Small Business Management, vol. 34, nº 1, p. 4658.

Rempel, J. K. et J. G. Holmes (1986), How do I trust thee ?, Psychology Today, vol. 20, nº 2, p. 28-34. 
Reynolds, P. D., S. M. Camp, W. D. Bygrave, E. Autio et M. Hay (2001), Global Entrepreneurship Monitor (GEM) - 2001 Summary Report, Global Entrepreneurship Monitor Report.

Rousillon, S. et J. Duval-Hamel (2006), Le stress des dirigeants : mythe, compétence-clé, risque pour l'entreprise? , Cahiers de recherche de l'EM Lyon Business School no ${ }^{\circ}$ 2006/3, EM Lyon Business School, France.

Sammut, S. (1998a), Comment aider les petites entreprises jeunes ?, Revue française de Gestion, $\mathrm{n}^{\mathrm{o}} 121$, p. 28-41.

Sammut, S. (1998b), Jeune entreprise - la phase cruciale du démarrage, Paris, L'Harmattan.

Sammut, S. (2003), L'accompagnement de la jeune entreprise, Revue française de Gestion, $\mathrm{n}^{\mathrm{o}}$ 144, p. 153-164.

Schaper, M. T., M. à. Campo et J. K. Imukuka (2005), The training and management development needs of micro-firms, Training \& Management Development Methods, vol. 19, no 2, p. 13-22.

Shaver, K. G. (1995), The entrepreneurial personality myth, Business and Economic Review, vol. $41, n^{\circ} 3$, p. 20-23.

Simon, M., B. Elango, S. M. Houghton et S. Savelli (2002), The successful product pioneer: maintaining commitment while adapting to change, Journal of Small Business Management, vol. 40, $\mathrm{n}^{\mathrm{o}} 3$, p. 187-203.

Simon, M., S. M. Houghton et K. Aquino (2000), Cognitive biases, risk perception, and venture formation:: How individuals decide to start companies, Journal of business venturing, vol. $15, n^{\circ} 2$, p. 113-134.

St-Jean, E. (2011), Mentor functions for novice entrepreneurs, Academy of Entrepreneurship Journal, vol. 17, $\mathrm{n}^{\mathrm{o}} 1$, p. 65-84.

St-Jean, E. et J. Audet (2012), The Role of Mentoring in the Learning Development of the Novice Entrepreneur, International Entrepreneurship and Management Journal, vol. 8, $\mathrm{n}^{\mathrm{o}}$ 1, p. 119-140.

St-Jean, É., J. Audet et N. Boucher (2007), L'apprentissage de l'entrepreneur réalisé dans le cadre d'une relation de mentorat : exploration des possibilités, $24^{e}$ Congrès annuel $d u$ $C C P M E / C C S B E$, Kelowna, C.B., $1^{\mathrm{e}}$ au 3 novembre.

St-Jean, E. et M. El Agy (2012), La motivation à être bénévole des mentors : un effet auprès des entrepreneurs accompagnés?, $2^{\text {ème }}$ Rencontre entre les acteurs des réseaux d'accompagnement et chercheurs, Montpellier, France, 19 janvier.

St-Jean, E. et S. Mitrano-Méda (2012), La formation des mentors d'entrepreneurs novices : une manière de s'assurer de la qualité de l'intervention de mentorat ? , 2ème Rencontre entre les acteurs des réseaux d'accompagnement et chercheurs, Montpellier, France, 19 janvier.

St-Jean, E. et M. Tremblay (2011), Opportunity Recognition With A Mentor: The Role Of The Entrepreneur's Learning Goal Orientation, Babson College Entrepreneurship Reseach Conference, Syracuse, N.Y., June $8^{\text {th }}-11^{\text {th }}$.

Sullivan, R. (2000), Entrepreneurial learning and mentoring, International Journal of Entrepreneurial Behaviour \& Research, vol. 6, n 3, p. 160-175.

Torrès, O. (2009), L'inaudible et inavouable souffrance patronale : le cas du licenciement en PME, in G. Lecointre (éd.) Le grand livre de l'économie PME, Gualino Éditeur.

Torrès, O. (2010), Quelques pistes provisoires de réflexion sur la santé des commerçants et artisans en France, 10ème Conférence Internationale Francophone en Entrepreneuriat et $P M E$, Bordeaux, France, 26-29 octobre. 
Ucbasaran, D., P. Westhead et M. Wright (2009), The extent and nature of opportunity identification by experienced entrepreneurs, Journal of Business Venturing, vol. 24, $\mathrm{n}^{\mathrm{o}} 2$, p. 99-115.

Valéau, P. (2006), L'accompagnement des entrepreneurs durant les périodes de doute, Revue de l'Entrepreneuriat, vol. 5, $\mathrm{n}^{\mathrm{o}}$ 1, p. 31-57.

Valéau, P. (2007), L'engagement des entrepreneurs : des doutes au second souffle, Revue Internationale PME, vol. 20, $\mathrm{n}^{\mathrm{o}}$ 1, p. 121-154.

Verzat, C. et C. Gaujard (2009), Expert, conseiller, mentor, confident ou tout à la fois ?, L'Expansion Entrepreneuriat, ${ }^{\circ}$ 2, p. 6-12.

Wanberg, C. R., J. Kammeyer-Mueller et M. Marchese (2006), Mentor and protégé predictor and outcomes of mentoring in a formal mentoring program, Journal of Vocational Behavior, vol. $69, n^{\circ} 3$, p. 410-423.

Wanberg, C. R., E. T. Welsh et S. A. Hezlett (2003), Mentoring Research: A Review and Dynamic Process Model, in J. J. Martocchio et G. R. Ferris (éds.), Research in Personnel and Human Resources Management, Oxford, U.K., Elsevier Science Ltd.

Waters, L., M. McCabe, D. Kiellerup et S. Kiellerup (2002), The role of formal mentoring on business success and self-esteem in participants of a new business start-up program, Journal of Business and Psychology, vol. 17, n 1, p. 107-121.

Wikholm, J., T. Henningson et C. M. Hultman (2005), Demand of mentoring among new starters, ICSB $50^{\text {th }}$ World Conference, Washington, DC.

Wolfe, J. et C. Jackson (1987), Creating Models of the Strategic Decision Making Process via Participant Recall: A Free Simulation Examination, Journal of Management, vol. 13, $\mathrm{n}^{\mathrm{o}} 1$, p. 123-134. 